\title{
Informed reasoning: repositioning of nitisinone to treat oculocutaneous albinism
}

\author{
Prashiela Manga and Seth J. Orlow \\ Ronald O. Perelman Department of Dermatology and Department of Cell Biology, New York University School of Medicine, New York, New York, USA.
}

\begin{abstract}
Oculocutaneous albinism (OCA) is a group of genetic disorders characterized by hypopigmentation of the skin, hair, and eyes. Affected individuals experience reduced visual acuity and substantially increased skin cancer risk. There are four major types of OCA (OCA1-OCA4) that result from disruption in production of melanin from tyrosine. Current treatment options for individuals with OCA are limited to attempts to correct visual problems and counseling to promote use of sun protective measures. However, Onojafe et al., reporting in this issue of the JCI, provide hope for a new treatment approach for OCA, as they demonstrate that treating mice that model OCA-1b with nitisinone, which is FDA approved for treating hereditary tyrosinemia type 1, elevates plasma tyrosine levels, and increases eye and hair pigmentation.
\end{abstract}

\section{Oculocutaneous albinism}

Melanin is a biopolymer synthesized from tyrosine. Upon synthesis, it is deposited in specialized organelles (melanosomes) in neural crest-derived melanocytes found in the skin, hair follicles, iris, uveal tract, and inner ear as well as in retinal pigmented epithelium (RPE) derived from the optic cup. Oculocutaneous albinism (OCA) denotes a group of common autosomal-recessive disorders that result from disruption of melanin synthesis. There are four major forms: OCA1, which is caused by mutations in the tyrosinase gene (TYR) (1); OCA2, caused by mutations in OCA2 (2); OCA3, caused by mutations in the tyrosinase-related protein 1 gene (TYRP1) (3); and OCA4, caused by mutations in the solute carrier family 45 , member 2 gene (SLC45A2) (4). OCA1 affects approximately 1 in 40,000 people worldwide and is the most prevalent form of OCA among individuals of mixed European descent. OCA2 affects 1 in 39,000 such individuals, with substantially higher prevalence in AfricanAmerican and Amerindian populations. The carrier rate of a pathogenic deletion of OCA2 in African-American newborns is estimated to be 1 in 235 (5); rates in some Amerindian populations reach 1 in 200 (6). OCA3 is rare, with notable frequency only in African populations (3). The inci-

Conflict of interest: In the past year, Seth J. Orlow served as a consultant to Astellas Pharmaceuticals, Bristol Myers Squibb, Galderma, Glaxo Smith Kline, Provectus Pharmaceuticals, and SkinMedica.

Citation for this article: J Clin Invest. 2011; 121(10):3828-3831. doi:10.1172/JCI59763. dence of OCA4 is not known; however, it appears to be more prevalent in Japanese populations (7).

OCAs are characterized by decreased or absent melanin in skin, hair, and eyes. In skin, melanin plays a central role in protection from UV-induced carcinogenesis. Thus, individuals with OCA are highly susceptible to skin cancers, particularly squamous cell carcinoma. During embryogenesis, melanin synthesis in the RPE affects development of the fovea and of adjacent retinal ganglion cells, which in turn regulate decussation of the nerve connecting the retina to brain at the optic chiasm. Numerous structural abnormalities ensue if melanogenesis is deficient. In the adult eye, melanin appears important for reducing light scatter and aiding acuity. Individuals with OCA consequently suffer from nystagmus, strabismus, photophobia, and loss of visual acuity that can be severe enough to render them legally blind (8).

The highly visible OCA phenotype most dramatic in individuals with OCA1, who have white hair, very pale skin, and light-colored irides - is a source of significant emotional and psychological stress. Visual insufficiencies are often misinterpreted as intellectual deficits in affected individuals, exacerbating feelings of alienation. A study of Nigerians with OCA2 found that they experienced alienation, avoided social interactions, and were less emotionally stable. Furthermore, affected individuals were less likely to complete schooling, find employment, and find partners (9).

\section{Melanin synthesis and its disruption in OCA}

Tyrosinase, a type I membrane protein, catalyzes the first and rate-limiting reaction during melanin synthesis. Unlike many membrane proteins that exit the ER rapidly, tyrosinase is retained for an unusually long period prior to transport to the Golgi, which suggests that it undergoes highly regulated/ complex early processing. Maturation of tyrosinase begins during translation, when the peptide is inserted into the ER membrane by Sec61, then bound by the folding chaperone BiP (also known as HSPA5) (Figure 1). Additional chaperones - calnexin, then calreticulin - subsequently bind the peptide to facilitate glycosylation. The foldase ER protein 57 (ERp57) is also recruited to the complex to catalyze disulfide bond formation after signal sequence cleavage. The processed peptide is then subject to the ER quality control system that ensures that only correctly folded proteins exit the ER. Correctly folded tyrosinase is transported to the Golgi, where it undergoes additional glycosylation. Misfolded tyrosinase is retained in the ER, undergoing cycles of folding and unfolding, until it is eventually targeted for proteasomal degradation (10).

Many OCA1-causing TYR mutations result in a protein that fails to fold and is therefore unable to exit the ER (11). Tyrosinase is also retained in the ER, due to unknown mechanisms, in OCA2 (12) and OCA3 (13), whereas in OCA4, tyrosinase exits the ER but is not correctly transported to the melanosome (14). We have previously demonstrated that ER retention of tyrosinase activates the unfolded protein response (UPR) (15), a highly conserved cell signaling cascade that increases the capacity of a cell to process misfolded proteins but can lead to apoptosis if ER stress is sustained.

The precise functions of the remaining OCA-related proteins (excepting tyrosinase) remain unclear. We have proposed that OCA2 mediates glutathione transport into the ER to promote disulfide bond formation during tyrosinase maturation (16) and that TYRP1 stabilizes tyrosinase 


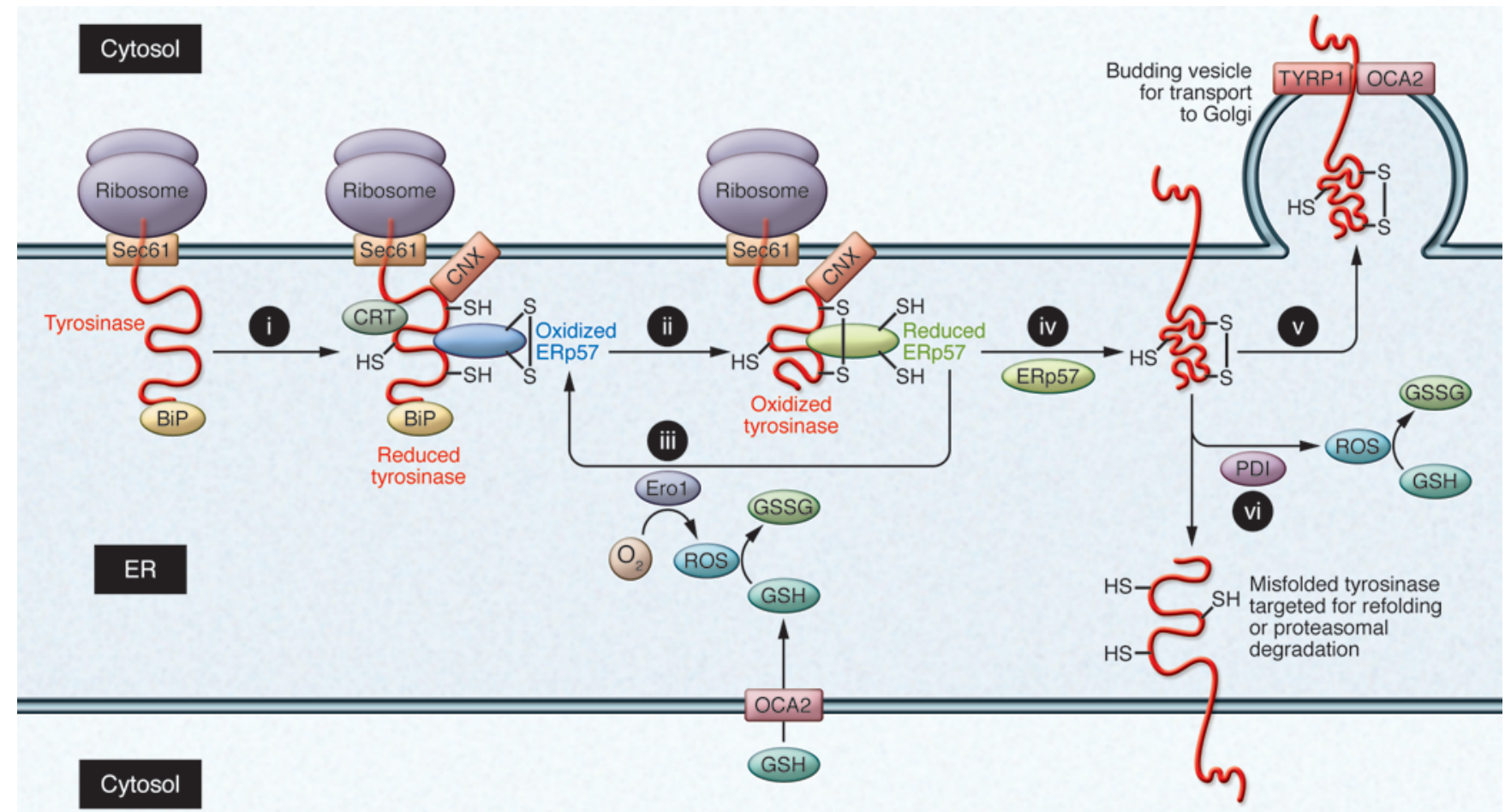

Figure 1

Synthesis and ER-based maturation of tyrosinase. Tyrosinase is synthesized on ribosomes and translocated into the ER membrane. (i) Tyrosinase is bound by the chaperones BiP, then calnexin (CNX), followed by ERp57 (ii) and calreticulin (CRT). Each chaperone is required to facilitate tyrosinase folding and glycosylation. In order to fold the peptide, protein disulfide isomerases (PDIs) such as ERp57 catalyze the formation of disulfide bonds. (iii) ERp57 is converted to a reduced state during catalysis. In order to recycle the protein, the ER oxidoreductin Ero1 oxidizes the PDI, generating ROS in the process. These ROS are detoxified by reduced glutathione (GSH), which is transported into the ER from the cytosol through a channel. Based on our studies (16), we hypothesize that the OCA2 protein serves as a GSH pump. (iv) ERp57 then catalyzes the isomerization of disulfide bonds to form a folded peptide. Correctly folded tyrosinase is released by the chaperones and then (v) recruited to a vesicle for transport to the Golgi. Recruitment may be facilitated by OCA2 and TYRP1. (vi) In the event that the protein is misfolded, disulfide bonds are removed by PDIs, which generates ROS that are removed by GSH, and tyrosinase is refolded. There are a limited number of protein refolding cycles, after which tyrosinase is transported to and degraded by proteasomes. GSSG, oxidized glutathione.

(17). Costin et al. have proposed a role for the OCA4 protein, SLC45A2, in transport of tyrosinase from Golgi to melanosomes, given that mutations in the SLC45A2 locus result in trafficking of functional tyrosinase to the plasma membrane (14).

\section{Improving tyrosinase folding and finding a treatment for OCA}

Individuals with OCA1 can carry TYR mutations that result in complete loss of tyrosinase activity (OCA-1A) or mutations that result in a protein with reduced activity at body temperature (OCA-1B). After postnatal molt, the hairs of mice carrying what is known as the Himalayan Tyr mutation, which generates a mutant protein with reduced tyrosinase activity at body temperature, are darker in cooler, acral body areas (as in Siamese cats). In vitro studies have shown that folding of temperaturesensitive tyrosinase produced by human
TYR mutations can be improved by culturing cells at temperatures of approximately $31^{\circ} \mathrm{C}(11)$. In the early 1960 s, long before the tyrosinase-encoding gene was identified, researchers found that hair bulbs from individuals with what we now call OCA-1A were incapable of making melanin when incubated at high concentrations of tyrosine, the substrate for tyrosinase, leading to OCA-1A (then termed tyrosinase-negative albinism). In contrast, incubation of hair bulbs from individuals with residual tyrosinase activity (which we now know include those with OCA-1B and OCA2 as well as other forms of OCA in which tyrosinase activity is diminished but not obliterated) in tyrosine causes pigment deposition, resulting in these forms being lumped together as tyrosinase-positive albinism. Topical application of tyrosine has not proven useful, presumably because of issues with permeation/penetration through skin, which acts as a barrier, to the melanocytes at the epidermal basal layer. Tyrosinase processing in cultured melanocytes can be greatly improved by tyrosine, DOPA (which is a cofactor for tyrosinase), and a variety of $\mathrm{pH}$-altering agents, including monensin and bafilomycin (18).

Building on these observations, Onojafe et al. report in this issue of the JCI on a potential treatment for OCA-1B (19). They were inspired by a side effect of sorts to make ingenious use of a drug, nitisinone, approved since 2002 to treat hereditary tyrosinemia type 1 (HT-1). Individuals with HT-1 have high levels of serum tyrosine as a result of deficiency of the enzyme fumarylacetoacetate hydrolase (FAH), the last enzyme in the tyrosine catabolism pathway. Somewhat counterintuitively, nitisinone raises serum tyrosine levels in individuals with HT-1 even further by blocking tyrosine catabolism one step upstream of FAH, acting as a competitive inhibitor of 


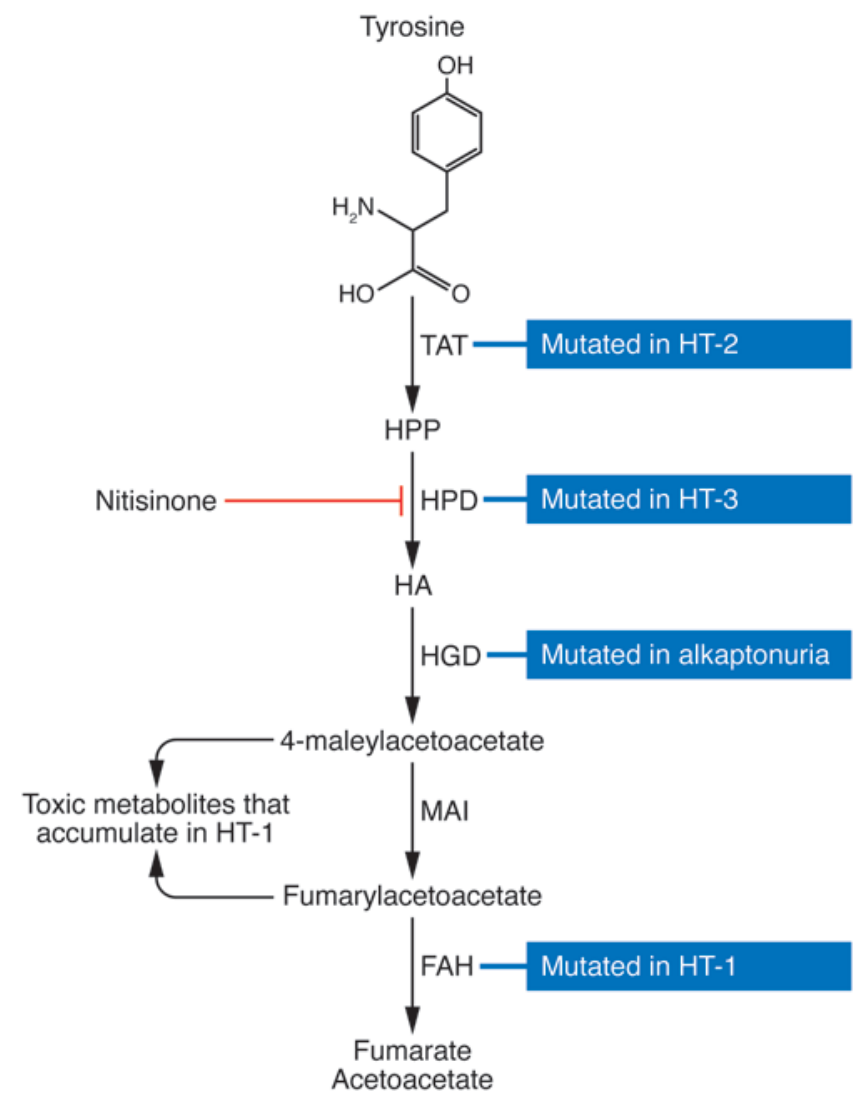

Figure 2

Tyrosine catabolism. Five enzymes catalyze the cascade that leads to the degradation of tyrosine, and dysfunction of these enzymes leads to diseases of variable severity (reviewed in ref. 21). Tyrosine is converted to 4-hydroxyphenylpyruvate (HPP) by tyrosine aminotransferase (TAT). Mutations of this enzyme lead to HT-2. HPP is converted to homogentisic acid (HA) by hydroxyphenylpyruvate oxidase (HPD), the target of nitisinone. HPD mutations result in HT-3. $\mathrm{HA}$ is converted to 4-maleylacetoacetate (by homogentisate 1,2-dioxygenase [HGD], mutations of which cause alkaptonuria) then fumarylacetoacetate (by malelylacetoacetate isomerase [MAI]). These metabolites are toxic and accumulate, leading to the symptoms common in HT-1 (due to mutations of FAH), which converts fumaryl acetate to fumarate and acetoacetate.

4-hydroxyphenylpyruvate oxidase (Figure 2) and thus preventing accumulation of the toxic intermediates of tyrosine breakdown that cause the severe symptoms of HT-1. Inspired by this side effect (unlike typical drug repositioning), Onojafe and colleagues reasoned that administration of nitisinone to albino mice might result in elevated levels of tyrosine in tissues including RPE and epidermis (19), where it could then act (as in the hair bulb test) to rescue tyrosinase, presumably as a substrate chaperone. Substrate chaperones have been proposed for the treatment of genetic disorders including cystic fibrosis and Gaucher, Fabry, Tay-Sachs, and Sandhoff diseases.

Consistent with their hypothesis, Onojafe and colleagues found that administration of nitisinone at a dosage 2-4 times higher than used to treat HT-1 increased fur and eye pigmentation in OCA-1B mice (19). Treatment had no effect in OCA-1A mice, presumably because their misfolded tyrosinase is incapable of binding tyrosine. Based on these observations, one might expect that correction of tyrosinase misfolding in humans can be achieved, resulting in increased pigmentation of skin and hair. This would offer significant improvement for individuals with OCA, in that they will benefit from increased melanin protection from UV-induced skin cancers and amelioration of the significant psychosocial impact of hypopigmentation.

Developmental defects due to lack of melanogenesis during embryogenesis are not expected to be correctable in an adult. Instead, correction would need to occur during the appropriate developmental window. To that end, Onojafe et al. gave nitisinone to pregnant OCA-1B mice and found that pigmentation was augmented in their offspring (19). While correction of developmental ocular defects remains to be proven, the augmented melanogenesis that they observed during gestation is highly encouraging. Since OCA-1B is a recessive disorder, parental carrier status is usually unknown until a first affected child is born. It is therefore unlikely that we will be able to treat women early enough during pregnancy to affect optic tract development, which is complete by $54-56$ days. Nitisinone treatment may thus be an option in second pregnancies or in cases where family history suggests a high chance of an affected pregnancy.

\section{Challenges for the future}

The challenge remains to develop similar approaches for other OCAs. We have previously demonstrated that tyrosine can improve tyrosinase folding in OCA2 melanocytes (20), which suggests that nitisinone deserves exploration in OCA2 models. This must be tempered with the knowledge that a proportion of tyrosinase in OCA2 is trapped in vesicles and fails to reach the melanosome. Furthermore, excess tyrosine increases tyrosinase expression and may exacerbate ER stress as a result of increased retention of the enzyme (also a possible complication in the treatment of other forms of OCA, including OCA-1B). While our prior studies showed that UPR activation caused by tyrosinase misfolding does not lead to apoptosis (15), a further tipping of the balance may lead to melanocyte death, although this awaits experimental testing.

The work of Onojafe et al. (19) represents a substantial leap forward toward the possible treatment of all forms of OCA. However, it also reminds us that in addition to rational crystallographic design and screening of large compound libraries, informed reasoning can identify promising therapeutics.

\section{Acknowledgments}

Supported in part by grant AR41880 from NIAMS, NIH.

Address correspondence to: Seth J. Orlow, Ronald O. Perelman Department of Dermatology, New York University School of Medicine, 560 First Ave, TH100, New York, New York 10016, USA. Phone: 212.263.5245; Fax 212.263.5819; E-mail: Seth.Orlow@nyumc.org. 
1. Oetting WS, King RA. Molecular basis of albinism: mutations and polymorphisms of pigmentation genes associated with albinism. Hum Mutat. 1999; 13(2):99-115.

2. Lee ST, Nicholls RD, Bundey S, Laxova R, Musarella M, Spritz RA. Mutations of the P gene in oculocutaneous albinism, ocular albinism, and Prader-Willi syndrome plus albinism. N EnglJ Med. 1994;330(8):529-534.

3. Manga P, Kromberg JG, Box NF, Sturm RA, Jenkins T, Ramsay M. Rufous oculocutaneous albinism in southern African Blacks is caused by mutations in the TYRP1 gene. Am J Hum Genet. 1997; 61(5):1095-1101.

4. Newton JM, et al. Mutations in the human orthologue of the mouse underwhite gene (uw) underlie a new form of oculocutaneous albinism, OCA4. Am J Hum Genet. 2001;69(5):981-988.

5. Durham-Pierre D, King RA, Naber JM, Laken S, Brilliant $\mathrm{MH}$. Estimation of carrier frequency of a $2.7 \mathrm{~kb}$ deletion allele of the $\mathrm{P}$ gene associated with OCA2 in African-Americans. Hum Mutat. 1996; 7(4):370-373.

6. Woolf CM, Dukepoo FC. Hopi indians, inbreeding, and albinism. Science. 1969;164(3875):30-37.

7. Inagaki $\mathrm{K}$, et al. Oculocutaneous albinism type 4 is one of the most common types of albinism in Japan. Am J Hum Genet. 2004;74(3):466-471.
8. Rachel RA, et al. Spatiotemporal features of early neuronogenesis differ in wild-type and albino mouse retina. J Neurosci. 2002;22(11):4249-4263.

9. Ezeilo BN. Psychological aspects of albinism: an exploratory study with Nigerian (Igbo) albino subjects. Soc Sci Med. 1989;29(9):1129-1131.

10. Wang N, Hebert DN. Tyrosinase maturation through the mammalian secretory pathway: bringing color to life. Pigment Cell Res. 2006;19(1):3-18.

11. Halaban R, Svedine S, Cheng E, Smicun Y, Aron R, Hebert DN. Endoplasmic reticulum retention is a common defect associated with tyrosinase-negative albinism. Proc Natl Acad Sci U S A. 2000;97(11):5889-5894.

12. Chen K, Manga P, Orlow SJ. Pink-eyed dilution protein controls the processing of tyrosinase. Mol Biol Cell. 2002;13(6):1953-1964.

13. Toyofuku K, Wada I, Valencia JC, Kushimoto T, Ferrans VJ, Hearing VJ. Oculocutaneous albinism types 1 and 3 are ER retention diseases: mutation of tyrosinase or Tyrp1 can affect the processing of both mutant and wild-type proteins. FASEB J. 2001;15(12):2149-2161

14. Costin GE, Valencia JC, Vieira WD, Lamoreux ML, Hearing VJ. Tyrosinase processing and intracellular trafficking is disrupted in mouse primary melanocytes carrying the underwhite (uw) mutation. A model for oculocutaneous albinism (OCA) type 4.
J Cell Sci. 2003;116(pt 15):3203-3212.

15. Manga P, Bis S, Knoll K, Perez B, Orlow SJ. The unfolded protein response in melanocytes: activation in response to chemical stressors of the endoplasmic reticulum and tyrosinase misfolding. Pigment Cell Melanoma Res. 2010;23(5):627-634.

16. Staleva L, Manga P, Orlow SJ. The pink-eyed dilution protein modulates arsenic sensitivity and intracellular glutathione metabolism. Mol Biol Cell. 2002;13(12):4206-4220.

17. Manga P, Sato K, Ye L, Beermann F, Lamoreux ML, Orlow SJ. Mutational analysis of the modulation of tyrosinase by tyrosinase- related proteins 1 and 2 in vitro. Pigment Cell Res. 2000;13(5):364-374.

18. Manga P, Orlow SJ. Inverse correlation between pink-eyed dilution protein expression and induction of melanogenesis by bafilomycin A1. Pigment Cell Res. 2001;14(5):362-367.

19. Onojafe IF, et al. Nitisinone improves eye and skin pigmentation defects in a mouse model of oculocutaneous albinism. J Clin Invest. 2011; 121(10):3914-3923.

20. Manga P, Boissy RE, Pifko-Hirst S, Zhou BK, Orlow SJ. Mislocalization of melanosomal proteins in melanocytes from mice with oculocutaneous albinism type 2. Exp Eye Res. 2001;72(6):695-710.

21. Scott CR. The genetic tyrosinemias. Am JMed Genet C Semin Med Genet. 2006;142C(2):121-126.

\title{
Viruses and human brain tumors: cytomegalovirus enters the fray
}

\author{
Cynthia Hawkins $^{1}$ and Sidney Croul ${ }^{2}$
}

\author{
${ }^{1}$ Division of Pathology, The Hospital for Sick Children, and 2Division of Pathology, University Health Network, Toronto, Ontario, Canada.
}

\begin{abstract}
Medulloblastoma is the most common malignant brain tumor in children. Overall survival rates have improved in recent years as a result of risk-stratified treatment regimens. However, medulloblastoma remains associated with substantial mortality, and survivors often experience debilitating neurological, endocrinological, and social sequelae as a result of treatment. Targeted and less toxic therapeutic strategies are therefore needed. In this issue of the JCI, Baryawno et al. report their findings that a large percentage of primary medulloblastomas and medulloblastoma cell lines are infected with human cytomegalovirus (HCMV) and suggest that targeting this virus could provide a new way to treat individuals with medulloblastoma.
\end{abstract}

Brain tumors account for $20 \%$ of all neoplasms in children and are the largest group of solid tumors that develop in childhood (1). Medulloblastoma is the most common malignant pediatric brain tumor, constituting $20 \%-25 \%$ of pediatric central nervous system neoplasms $(2,3)$. Its incidence is estimated at 2-6 cases per million children per year, with approximately 540 new cases diagnosed annually in the United States $(2,4)$. Medulloblastoma typ-

Conflict of interest: The authors have declared that no conflict of interest exists.

Citation for this article: J Clin Invest. 2011; 121(10):3831-3833. doi:10.1172/JCI60005. ically arises in the midline cerebellum, in the region of the mid- and inferior vermis. Current treatment includes surgery, craniospinal irradiation, and chemotherapy. Overall survival for all medulloblastoma patients is roughly $50 \%-60 \%$ in population-based studies (5). However, as we have gained a better understanding of clinical risk factors, the incorporation of patient stratification in larger, multi-institutional studies has resulted in improved survival. Currently, there are three major treatment strategies for medulloblastoma patients based on their clinical status. For patients younger than 3-5 years, treatments are aimed at maximizing survival while avoid- ing radiation $(6,7)$. Older patients are stratified by the extent of resection and metastatic status. Patients with metastatic disease or a less-than-optimal resection are classified as high-risk and are treated with high doses of craniospinal irradiation (36-39 Gy) and aggressive chemotherapy. Patients with totally, or near-totally, resected, non-disseminated disease are designated average-risk. This is the most prevalent group, and these patients are treated with a combination of lower-dose irradiation and chemotherapy. This clinical stratification has resulted in higher cure rates for all groups, with $80 \%$ of average-risk patients reaching progression-free survival at 5 years $(8,9)$. Despite the improved survival, there still remains a substantial amount of mortality associated with medulloblastoma, and survivors often experience neurological, endocrinological, and social sequelae as a result of treatment. Thus, many researchers are seeking to develop new, more targeted and less toxic therapeutic strategies. In this issue of the JCI, Baryawno et al. describe a potential novel therapeutic strategy for medulloblastoma 